\title{
A First Response Microcontroller Based Digital Radiation Survey Meter Using SCINTILlation Detector
}

\author{
M.A.A. Mashud ${ }^{1}$, S.M.S. Hossain ${ }^{2}$ M.S. Hossain ${ }^{3}$, M.A. Razzaque ${ }^{1}$ and \\ M.S. Islam ${ }^{4}$ \\ ${ }^{1}$ Department of Applied Physics, Electronics \& Communication Engineering, \\ Islamic University, Kushtia-7003, Bangladesh \\ ${ }^{2}$ Department of Physics, Govt. B.L. College, Daulatpur, Khulna, Bangladesh \\ ${ }^{3}$ Department of Computer Science \& Engineering, Islamic University, Kushtia, \\ Bangladesh \\ ${ }^{4}$ Department of Medical Physics \& Biomedical Engineering, Gono Bishwabidyalay, \\ Savar, Dhaka, Bangladesh
}

\begin{abstract}
This paper presents the microcontroller based advanced technique to design and development of a portable radiation survey meter to measure low level gamma radiation using NAI(T1) scintillation detector. A scintillation detector was used as radiation detector and a microcontroller PIC16F876 was used to control the function of the developed system. The microcontroller generated square wave frequency at specified pulse width to produce high voltage $(+1200 \mathrm{~V})$ and regulates it. The high voltage was required to activate the scintillation detector. Preamplifier and amplifier were designed to make the detector signal for the further amplification. Microcontroller senses the pulses from the amplifier output and processes data by software and displays the results. The microcontroller was programmed using a high level programming language ' $C$ ' with PCWH compiler.
\end{abstract}

\section{KEYWORDS}

Gamma ray, Scintillation detector, NaI (T1), PIC16F876, PCWH, Digital and Portable

\section{INTRODUCTION}

Scintillation radiation survey meter is useful for measuring low-level gamma-ray in site to locate the heavy minerals and assess the adequacy of radioactive materials. It is being widely used in medical science applications and by the Geologists to detect Gamma ray from radioactive nuclei and to determine their energies. Radioisotopes and radiation instruments are being used in industries, agriculture, food processing, medicine, geologist, diagnostics and research laboratories. Radiation is very risky and seriously harmful for workers, public and to the environment, which may arise from these applications, have to be assessed and controlled.

Several radiation monitoring systems are designed locally in Bangladesh. A portable radiation survey meter using GM detector is explained in S. Islam et.al [1]. However this option cannot obtain a statistical analysis of digitally stored data, or any other form of analysis. The sensitive radiation survey meter as $\beta-\gamma$ radiation monitor using scintillation detector $\mathrm{NaI}(\mathrm{TI})$ is explained in

DOI: $10.5121 /$ ijci.2014.3502 
International Journal on Cybernetics \& Informatics (IJCI) Vol. 3, No. 5, October 2014

S. Islam et.al [2]. The above two systems are fully analog and a large number of components were used. However these systems are quite costly and complex in design. A PC based data acquisition system is explained in M.A.A. Mashud et.al [3]. A microcontroller based portable surface contamination monitor is explained in M. A. A. Mashud et.al [4]. In this design PIC16F676 microcontroller and GM detector has been used to detect the Beta-Gamma radiation from radioactive sources or environment. The response of this system is slightly slow to detect the low-level Gamma radiation.

We developed an alternative approach: a first response microcontroller based digital radiation survey meter. The developed system used scintillation detector that measures the low-level Gamma radiations. The analog outputs of the detector will be analyzed and converted to digital data using a microcontroller. Using readily available components and simple circuitry, the system is portable, low-cost, simple in design and fully digital.

This remainder of the paper is organized as follows: In section II describes the design consideration of this system with block diagram and circuit diagram. In section III describes the flowchart of the system program. The experimental results is describes in section IV. The last section describes the conclusion.

\section{DESIGN CONSIDERATION}

The main part of the proposed system is microcontroller. The system is consisting with: the detector circuit, high voltage power supply circuit, amplifier circuit, microcontroller and display circuit. The regulated high voltage $1200 \mathrm{~V}$ is generated from the system power supply $9 \mathrm{~V}$ battery. The positive terminal of the high voltage power supply is connected to the anode of the detector and the ground is connected to the cathode. When the gamma radiation energy incident on the detector, photon produced in the scintillator. These photon produce electron from photo cathode and dynode chain amplified or multiply the electrons and develops a negative going pulse. This pulses passes to the pre-amplifier circuit, which amplifies the input signal ten times at $180^{\circ}$ out of phase and amplifies the signal five times by the amplifier. After amplification, this signal passes to the microcontroller and processes data by software. The block diagram and the circuit of the proposed system are shown in Figure 1 and Figure 2 respectively.

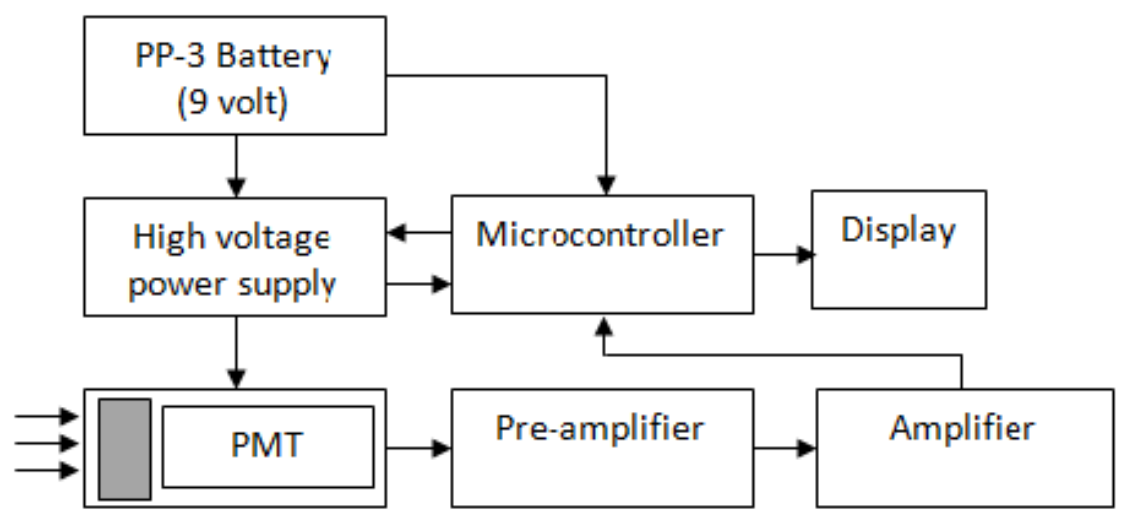

Figure 1. Block diagram of the proposed system 
International Journal on Cybernetics \& Informatics (IJCI) Vol. 3, No. 5, October 2014

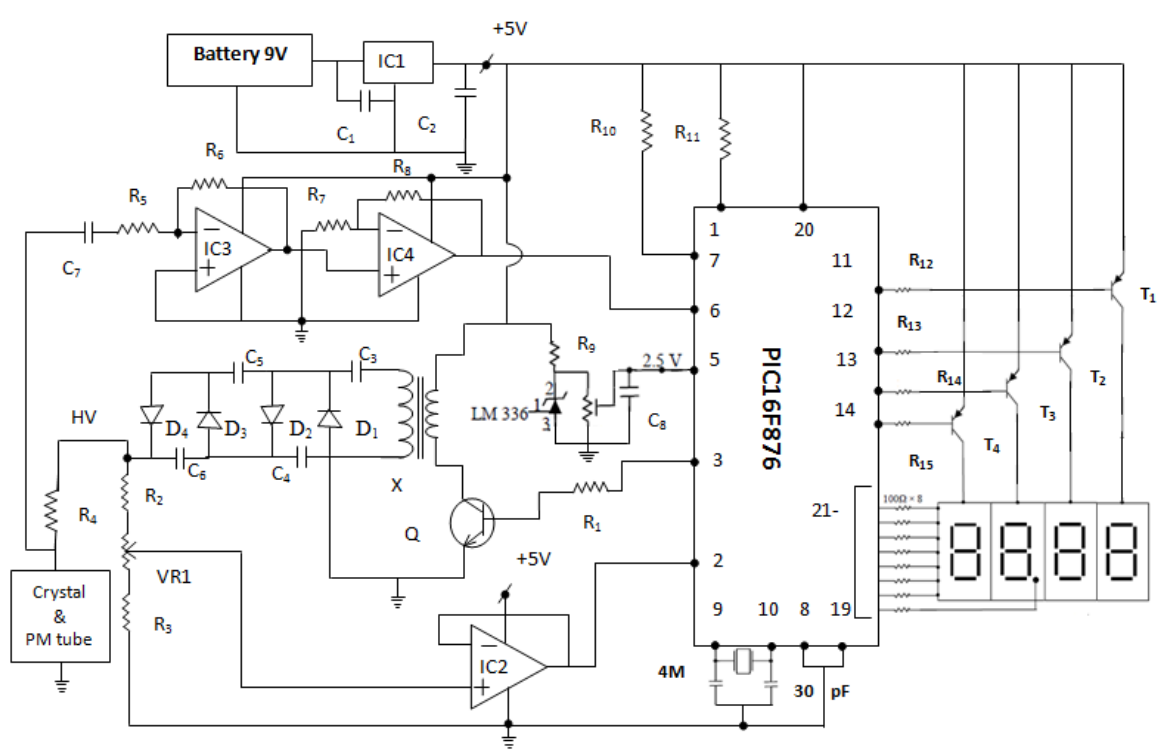

Figure 2. Circuit diagram of the proposed system

\subsection{Low Voltage Power Supply}

A low voltage de power supply is essential to power the microcontroller as well as other electronic components [5]. A 9V, PP-3 battery is used as a power for this portable instrument. To active the microcontroller a positive voltage regulator is used in this design for fixed $+5 \mathrm{~V}$ output voltage. And LM 336 is used to produce $2.5 \mathrm{~V}$.

\subsection{High Voltage Power Supply}

A regulated high voltage power supply is used to activate or bias the scintillation detector. The high voltage power supply unit consists of the following circuits: pulse generation and driver circuit, voltage quadoublers circuit, sampling circuit, error amplifier circuit [6]. In pulse generation circuit unipolar pulse train is generated from microcontroller and drives this pulse to a ferrite core step-up transformer through transistor driver circuit. Voltage sampler circuit samples the output voltage continuously to monitor variations from the desired value, up and down.

\subsection{Detector Circuit}

The output pulse height of the detector depends on the energy of the radiations under measurement and also on the biasing voltage of the detector. This unit consists of resistor $\mathrm{R}_{4}$ and scintillation detector [7].

\subsection{Pre-Amplifier}

An inverting 10 times gained pre-amplifier consists of CA3140 op-amp and resistors R5, R6. Resistor R5 is directly coupled to the coupling capacitor C7 of the detector. This circuit amplifies the signal produced by the detector due to the passes of photons into the detector; also it increases the voltage pulse by a small amount. The output of the pre-amplifier is $180^{\circ}$ out of phase compared to the input signal. The function of this amplifier [8] is to provide a voltage pulse whose height must be proportional to the total charge collected. The charge collection time must be equal to the rise time of the pulse. 


\subsection{Amplifier}

The amplifier circuit consists of $\mathrm{R}_{7}, \mathrm{R}_{8}$ and IC4. It is a non inverting amplifier circuit which amplifies five times in the same phase of the pre-amplifier output signal [9]. The output is in phase to the input signal. Amplifier output is connected to the microcontroller.

\subsection{Microcontroller Unit}

In our design a PIC16F876 programmable microcontroller is used. This microcontroller is very powerful with 200 nanoseconds instruction execution. It is very easy to program since it has only 35 single word instructions with CMOS FLASH. It's 8-bit microcontroller packs Microchips's powerful Programmable Interface Controller (PIC) architect into a 28-pin package and is compatible with the PIC16C5X, PIC12CXXX and PIC16C7X devices [10,11]. The Console Command Processor (CCP) of PIC microcontroller is capable of capture/compare/pulse-width module and also detect rising or falling edges every four or 16 pulses [12]. The features of PIC16F876 microcontroller are: 256 bytes of electrically erasable programmable read-only memory (EEPROM) or data memory, self programmble, 5 channels of 10-bit Analog-to-Digital (A/D) converter, 2 additional timers, 2 capture/compare/PWM functions, the synchronous serial port can be configured as either 3-wire Serial Peripheral Interface (SPI) or the 2-wire InterIntegrated Circuit (IIC) bus and a Universal Asynchronous Receiver Transmitter (USART). All of these features make it ideal for an advanced level A/D applications in automotive, industrial appliances and consumer applications.

\section{SYSTEM Program}

The system program has been developed for controlling the whole system. The program is divided into different sub routines and main routines. The compiler PCWH is used to develop the system program $[13,14,15]$. The flow chart of the system program is depicted in Figure 3.

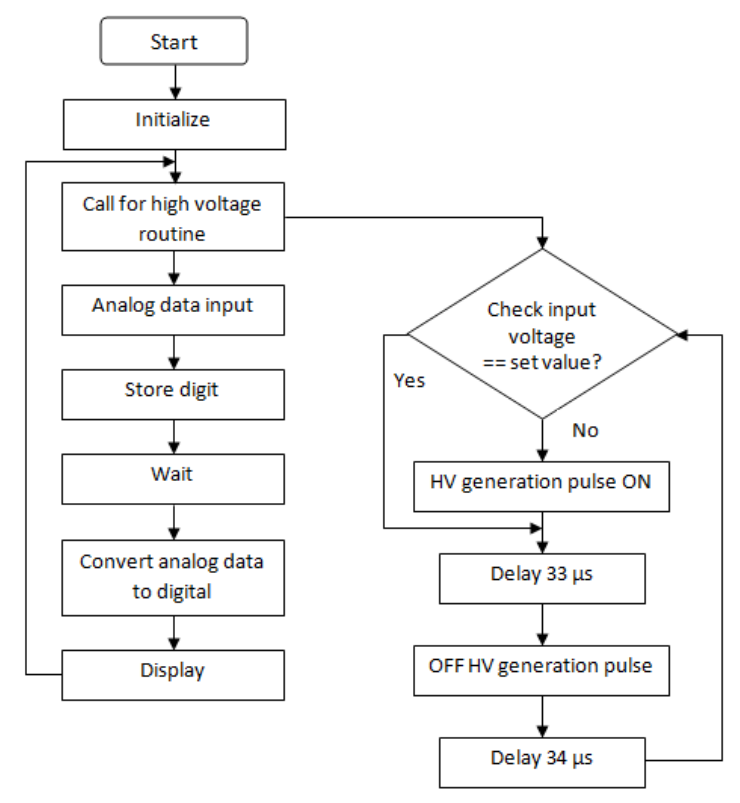

Figure 3. Flowchart of the system program 


\section{RESULTS AND DISCUSSION}

The system was developed and tested successfully. We found its strong performance. The system employed a local, low-cost PIC16F876 microcontroller. For avoiding the complexity of external comparator circuit and external A/D converter circuit, we used its internal comparator and A/D converter of the microcontroller. Thus the design complexity reduces and the system becomes portable and readable. The overall function depends on the developed software. The circuit design is simple and compact.

For biasing the microcontroller a strong $+5 \mathrm{~V}$ power supply was required. So we had designed a highly regulated $+5 \mathrm{~V}$ power supply. The battery voltage was varied from 9 volt to 5.5 volt but the output was remains unchanged to 5 volt DC. Figure 4 shows the waveform of low voltage power supply unit.

Besides a fixed voltage 2.5 volt is required as a reference voltage of the microcontroller which is connected to the pin 5 of the microcontroller. Here we have used LM336 IC which produces 2.5 volt. The output waveform of this unit is shown in Figure 5.

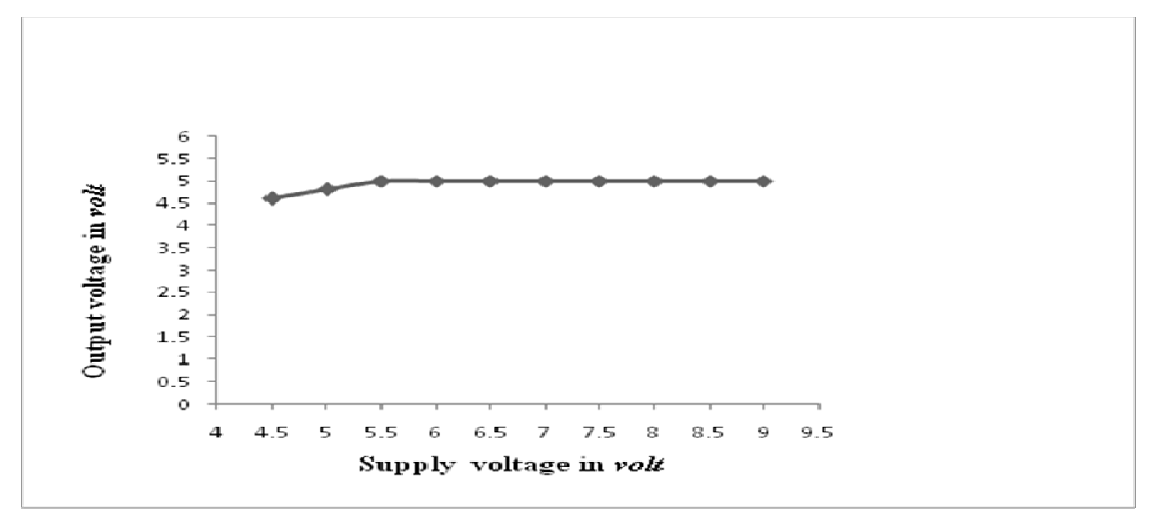

Figure 4: Output of IC1

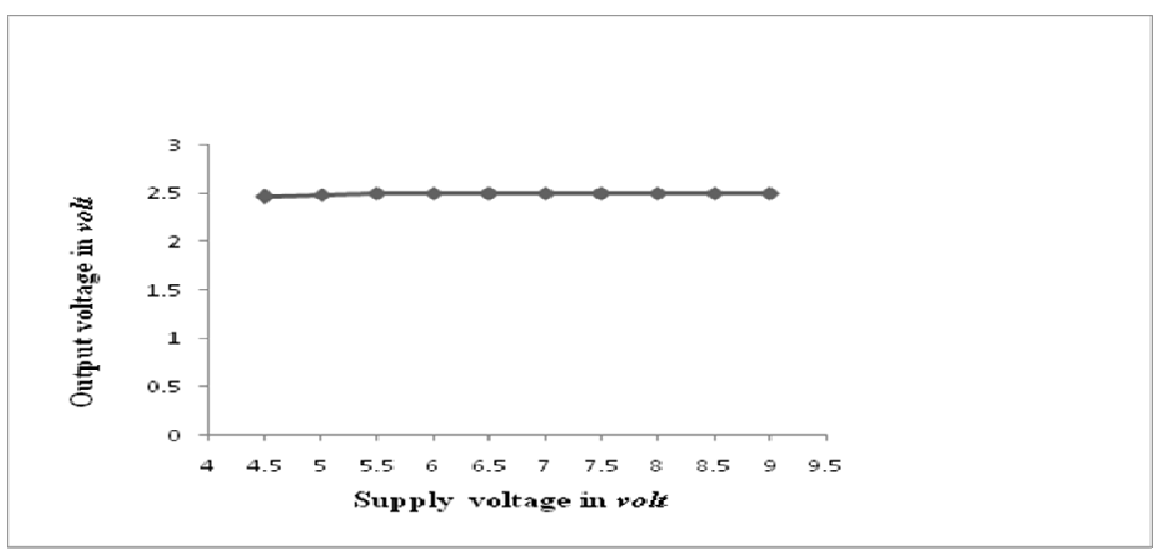

Figure 5: Output of LM336 


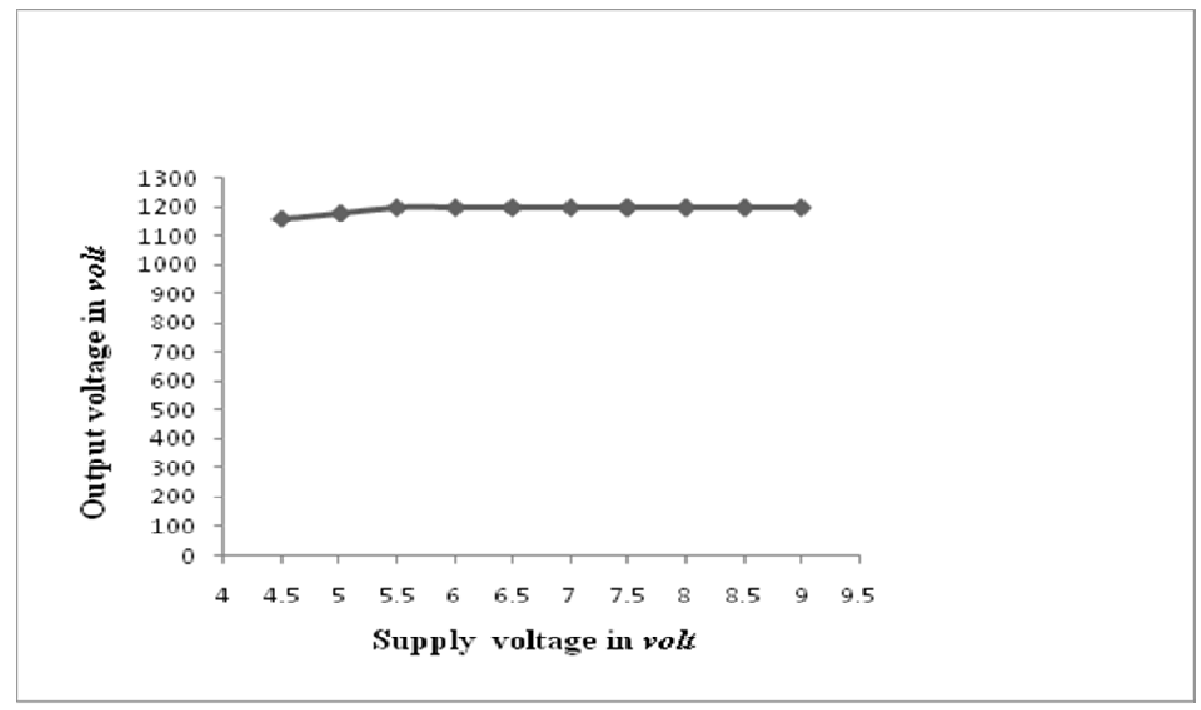

Figure 6: High voltage power supply

The regulated high voltage power supply unit was designed and developed successfully and it shows good performance. We have employed a locally available low-cost components, internal comparator and internal ADC. So, the software development and circuit design becomes very simple and small in size. We have used internal clock to avoid external circuit. In our proposed system, the output of the sampling circuit continuously sends the signal to the input port of the microcontroller. The microcontroller receives the corresponding voltages and internally compare with the reference value. When the microcontroller sensing input voltage lies between 0V to $2.4 \mathrm{~V}$, the microcontroller is so programmed that it produces a square wave of $15 \mathrm{KHz}$. When the microcontroller input sensing voltage over $2.4 \mathrm{~V}$, the microcontroller turns off the frequency generation. The frequency $15 \mathrm{KHz}$ is chosen because we have used ferrite core transformer that small in size. Figure 6 indicates the output of high voltage power supply unit. We observed that the output voltage remains unchanged i.e. fixed at $+1200 \mathrm{~V}$ dc since the input varies from 9 volt to 5.5 volt. This high voltage power supply is applicable for scintillation detector.

Each output pulse from the detector was counted. Figure 7 depicts the counts per second that represents the linearity graph of the developed system. The counts/second of the GM tube gives an approximation field of the radiation. Contamination linearly varies with count/s

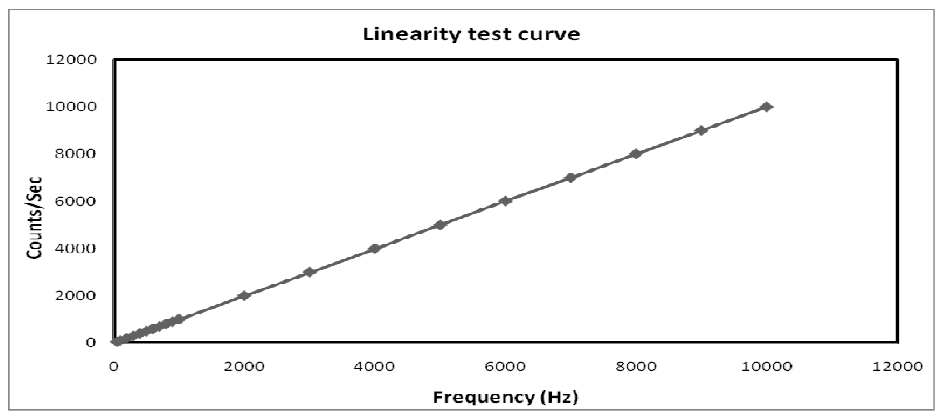

Figure 7. Linearity graph of the designed system 
International Journal on Cybernetics \& Informatics (IJCI) Vol. 3, No. 5, October 2014

The instrument underwent thorough tests, with satisfactory results. The system was calibrated using a radiation source from SSDL. The readings of the system were compared with the Canberra Nim Counter is shown in Figure 8. From the graphs, it seems that the developed system is sound better.

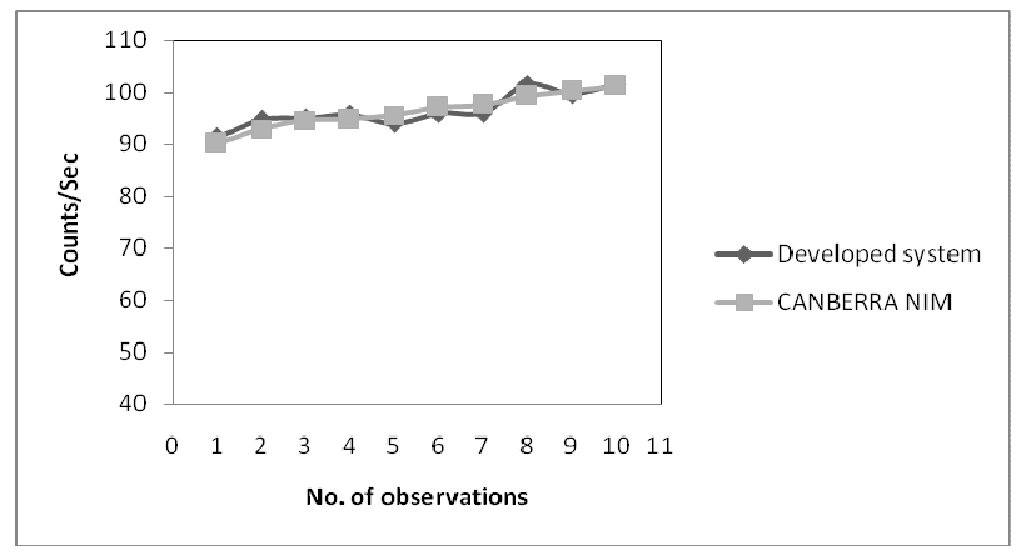

Figure 8: Comparison graph between developed system and CANBERRA NIM counter

The new technique for low-level Gamma radiation measurements appears technically simpler, industrially applicable, and potentially more accurate than conventional monitoring of Gamma radiation by aspiration and testing with performance. The technical specifications of the developed system are provided in Table 1 .

Table 1: Technical specifications of the developed system

\begin{tabular}{|c|c|c|}
\hline Feature & \multicolumn{2}{|l|}{ Specification } \\
\hline Power supply & \multicolumn{2}{|l|}{9 Volt PP-battery } \\
\hline High voltage & \multicolumn{2}{|l|}{1200 volt dc } \\
\hline Display & \multicolumn{2}{|c|}{ Four 7-segment display } \\
\hline Dose rate range & \multicolumn{2}{|l|}{ 0000-9999 cps } \\
\hline Response time & \multicolumn{2}{|l|}{ Nanoseconds } \\
\hline Power consumption & \multicolumn{2}{|l|}{$345 \mathrm{~mW}$} \\
\hline \multirow[t]{4}{*}{ Detector } & \multicolumn{2}{|c|}{ Model: PGS-3 of Technical Associates, U.S.A } \\
\hline & Crystal material & $\mathrm{Na}(\mathrm{T} 1)$ \\
\hline & Crystal diameter & 1.00 inch. \\
\hline & Crystal thickness & 1.00 inch. \\
\hline
\end{tabular}

\section{Conclusions}

Now-a-days the cost of electronic equipment is low, but the cost of nuclear equipment is high. In the scientific world the rapid development of microelectronics, all useful components and instruments becomes inexpensive. The proposed system has been presented that incorporates the uses of microcontroller and sensors in developing a low-cost, high-accuracy radiation monitoring system, avoiding external analogue and digital components. Its costs approximately U\$ 1000 for its fabrication, whereas the price of the similar instrument in the international market is not less than U\$ 3000. The proposed system has been completed and tested through a series of experiments and the results have proven the sound accuracy and reliability of the system. The 
International Journal on Cybernetics \& Informatics (IJCI) Vol. 3, No. 5, October 2014

features of the system has compared with the presently available different types of radiation monitoring system and shows that the proposed system is of better choice due to the cost, portability and data logging capability.

\section{REFERENCES}

[1] S. Islam, H. Rahman and M. Aziz (1988) ; Elect. Engig. Research Bulletin. Vol. 4, No. 1, pp.1.

[2] S. Islam, A. Kiber and M. A. Taher (1989); Nuclear Science and Applications, Vol. 1, No. 2, pp.12.

[3] M. A.A. Mashud, M.S. Hossain, M.N .Islam and Md. Serajul Islam (2013); mecs-press: I.J. Image, Graphics and Signal Processing. Issue.7, pp.34.

[4] M. A. A. Mashud, M. R. A. Bhuiyan, M. A. Masud and Md. Serajul Islam (2011); ULAB Journal of Science and Engineering, Vol.2, pp.42.

[5] M. A.A. Mashud, S.C. Barman, M.R.A. Bhuiyan and Md. Serajul Islam (2013); mecs-press: I.J. Intelligent Systems and Applications. Issue.10, pp.35.

[6] M. A.A. Mashud, M.R.A. Bhuiyan, and Md. Serajul Islam (2013); Journal of Physics Studies. Vol.19, pp.71.

[7] King Henry's Drive (1992); “Geiger-Muller Tubes Data book” Centronic Limited, New Addington.Survey,England, pp.31-32.

[8] Robert F. Coughlin and Frederick G. Driscoll (2003); “Operational Amplifier and Linear Inegratted Circuits"Prentice-Hall of International, Fifth Edition.

[9] Ramakant A. Gaykaward (2001); “Op-amps and Linear Integrated Circuits" Prentice-Hall India, Third Edition.

[10] M. A. Mazidi, R.M. Kinlay \& D. Causey (2008); "PIC Microcontroller", Prentice Hall Inc., pp.24.

[11] J. B. Peatman (1997); "Design with PIC microcontroller", Prentice Hall Inc., pp2.

[12] "Microchip Data sheet(2010)" PIC16F876.shtml accessed on 5.8.2010.

[13] PCWH Compiler, version 3.43

[14] E Balagurusamy (2001); "Programming in ANSI C"TATA Mc-GRAW HILL, Second Edition.

[15] M. A.A. Mashud, M.S. Hossain, M.N. Islam M.S. Islam and M. Shahinuzzaman (2012); Journal of Physics Studies. Vol.2, pp.15.

\section{Authors}

Md. Abdullah-Al-Mashud was born on Nov.15, 1980 in kushtia, Bangladesh. He received the B.Sc (Hons) degree and M.Sc degree in Applied Physics, Electronics and Communication Engineering (APECE) from Islamic University, Kushtia, Bangladesh in 2003 and 2004 respectively. He works as a faculty member in the department of APECE, Islamic University, Bangladesh. His current interest is microprocessor / microcontroller applications in control, automation, medical instruments, environmental monitoring, low cost electronic systems, Medical Image Processing. His work has produced 13 peer-reviewed scientific International and National Journal papers. He has published 06 papers in National and International Conferences.

S.M. Sajjad Hossain Was born on Oct. 30, 1973 in Satkhira, Bangladesh. He received the M.Sc degree in Physics from Rajshahi University, Bangladesh. He is an Assistant professor in the Department of Physics, B.L. College, Khulna. His work has produced 05 peer-reviewed scientific papers

M. Shamim Hossain was born on Oct. 21, 1982 in kushtia, Bangladesh. He received the B.Sc (Hons) degree and M.Sc degree in Computer Science and Engineering (CSE) from Islamic University, Kushtia, Bangladesh in 2006 and 2007 respectively. He works as a Assistant Professor in the department of CSE, Islamic University, Bangladesh. His current interest is microprocessor / microcontroller based system design and wireless networking. His work has produced 12 peer-reviewed scientific International Journal papers.
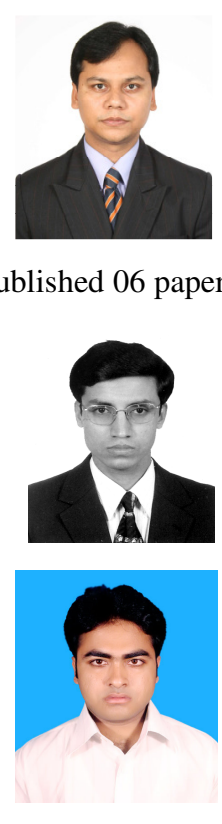
Md. Abdur Razzaque was born in May. 10, 1976 in Meherpur, Bangladesh. He received the M.Sc degree in Applied Physics, Electronics and Communication Engineering (APECE) from Islamic University, Kushtia, Bangladesh in 2000. He works as a Assistant Professor in the department of APECE, Islamic University, Bangladesh. His current interest is signal processing, Intelligent Controls and Mobile telecommunications. His work has produced 02 scientific 1 Journal papers.

Prof. Md. Serajul Islam was born in Panchagar, Bangladesh. He received the M.Sc degree in Physics from Rajshahi University, Bangladesh. He was a Chief Scientific Officer and Director in the Institute of Electronics, AERE, Atomic Energy Commission, Savar, Bangladesh. Now he is a professor in the Department of Medical Physics and Biomedical Engineering, Gono Bishwabidyalay. Saver, Bangladesh. His work is design, development and analysis of electronic instruments and reactor control. His work has produced nearly 50 peer-reviewed scientific papers and 02 patents.
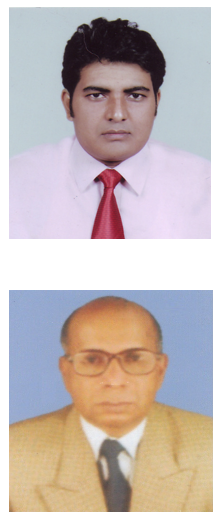